\title{
APROVEITAMENTO DE RESÍDUOS DE PAINÉIS DE MADEIRA GERADOS PELA INDÚSTRIA MOVELEIRA NA PRODUÇÃO DE PEQUENOS OBJETOS ${ }^{1}$
}

\author{
Luciana Barbosa de Abreu², Lourival Marin Mendes ${ }^{3}$ e José Reinaldo Moreira da Silva ${ }^{3}$
}

\begin{abstract}
RESUMO - Os painéis reconstituídos à base de madeira ganharam espaço antes ocupado pela madeira maciça, o que tem gerado volume expressivo de resíduos. A indústria moveleira contribui para a geração desses resíduos, que se apresentam como alternativa viável para a exploração de sua aplicação na produção artesanal de pequenos objetos. Este trabalho teve por objetivo produzir pequenos objetos com painéis e com mistura de resíduos de painéis de madeira, a fim de comparar suas qualidades. A partir de 10 projetos gráficos, três artesãos confeccionaram 10 pequenos objetos, utilizando painéis de MDF (Medium Density Fiberboard), OSB (Oriented Strand Board) e compensado. Cada artesão produziu nove exemplares de cada objeto. Um quarto artesão confeccionou os mesmos objetos com resíduos de três empresas do polo moveleiro da cidade de Ubá, MG, com três repetições, sendo comparados os atributos de qualidade dos objetos. Concluiu-se que todos os painéis são adequados para a confecção dos objetos e alguns se destacam em relação a outros, conforme o atributo avaliado. Os objetos confeccionados com a mistura de resíduos de painéis de madeira tiveram acabamento uniforme e não apresentaram diferenças significativas em relação aos produzidos com painéis com relação aos atributos funcionalidade, peso, proporção e simetria. A mistura de resíduos de diferentes painéis influenciou negativamente a avaliação dos atributos acabamento, beleza e cor. Um objeto produzido a partir de resíduos do mesmo painel pode ser mais harmonioso e atrativo. É tecnicamente viável a produção de pequenos objetos a partir de resíduos de painéis de madeira vindos de indústrias moveleiras.
\end{abstract}

Palavras-chave: Painéis de madeira, resíduos e pequenos objetos de madeira.

\section{SMALL OBJECTS MANUFACTURED WITH WOOD PANEL RESIDUES GENERATED BY THE FURNITURE INDUSTRY}

\begin{abstract}
Wood residue panels have been used in substitution of solid wood. As a consequence, the volume of residues has been increasing day-by-day. The furniture industries are the major generators of these residues. Their use as raw material for the production of handmade small objects emerges as an alternative to use the economic potential of this type of residue. The objective of this work was to produce small wood objects using boards and mixed board residues to compare their quality. At first, three workers were selected and supplied with MDF, OSB and plywood boards, as well as graphic projects of 10 small objects. Each worker produced 9 samples of each object. At a second turn, one worker was selected to produce the same 10 objects with three samples, mixing residues from three furniture industries of Ubá, MG. The quality attributes of the objects were evaluated and compared. It was concluded that all the wood boards used are adequate to produce the 10 objects but some boards are better, depending on the attribute evaluated. The objects manufactured with mixed woodboard residues presented similar finishing attributes and no significant differences from the objects produced with wood boards in the following attributes: functionality, weight, proportion and symmetry. Mixing residues of different boards affected the evaluation of the attributes: finishing, beauty and color. An object made from residues of the same board can be more attractive. It is technically feasible to produce small objects using residues of wood panels generated by the furniture industry.
\end{abstract}

Keywords: Wood boards, residues and small wood objects.

\footnotetext{
${ }^{1}$ Recebido em 11.09.2007 e aceito para publicação em 26.01.2009.

${ }^{2}$ Programa de Pós-Graduaçãoem Ciência e Tecnologia da Madeira da UniversidadeFederal de Lavras (UFLA). E-mail: <luarquiteta@ufla.br>.

${ }_{3}^{3}$ Departamento de Ciências Florestais da UFLA. E-mail: <lourival@ufla.br> e <jreinaldo@ufla.br>.
} 


\section{INTRODUÇÃO}

Hoje em dia, a tradicional madeira maciça vem sendo substituída por vários tipos de painéis, em virtude da relação preço/desempenho e da crescente conscientização da sociedade a respeito da exploração indiscriminada de reservas florestais (TOMASELLI e DELESPINASSE, 1997). Cresce a disponibilização de resíduos gerados pelas indústrias de base florestal, que, geralmente, incineram-nos ou descartam em locais impróprios (BRAND et al., 2004).

Os resíduos de painéis de madeira têm grande potencial econômico, ainda pouco explorado. Uma alternativa é o seu aproveitamento como matéria-prima na confecção artesanal de pequenos objetos. Essa idéia vem ao encontro do sentimento atual favorável pela adoção de materiais que provoquem o mínimo impacto ambiental.

Painéis reconstituídos à base de madeira são produtos que passam por um processo de redução e montagem. Entre alguns dos mais utilizados pelas indústrias madeireiras, podem-se citar o compensado laminado, o medium density fiberboard (MDF) e o oriented strand board (OSB). O produto formado por várias lâminas de madeira entrecruzadas, unidas perpendicularmente umas às outras, por meio de resina sintética ou outro tipo de adesivo, e submetidas à colagem sob prensagem aquecida, é denominado compensado laminado. Por sua vez, o OSB é um painel composto de partículas alongadas, que medem, geralmente, de 80 a $150 \mathrm{~mm}$ de comprimento por $25 \mathrm{~mm}$ de largura. Já o MDF é composto de fibras lignocelulósicas prensadas, apresentando estrutura homogênea e menos anisotrópica, o que lhes proporciona melhor capacidade de usinagem de bordas.

Os pequenos objetos de madeira são conhecidos no setor madeireiro pela sigla POM. Os POM podem ser agrupados em artigos domésticos de caráter utilitário, de caráter decorativo, de uso pessoal, brinquedos e complementos de outros produtos, entre outros. Eles apresentam propriedade mercadológica interessante em relação aos demais produtos de madeira, pois não sofrem exigência quanto à espécie de madeira. Se o produto atende às necessidades básicas de sua função, a espécie de madeira com a qual ele é confeccionado não interfere na sua aquisição (STERNADT, 2002). Pequenos objetos confeccionados com resíduos e apresentados como "reaproveitados" podem difundir os painéis de madeira e valorizar o trabalho artesanal.
Já é comum encontrar pequenos objetos de painéis de madeira no mercado de artesanato, entretanto a grande maioria é proveniente de painéis comprados pelos artesãos. Notou-se que alguns artesãos já buscavam trabalhar com material alternativo e reciclado, pois percebem como pode ser vantajoso ganhar ou arrematar, por baixo valor, peças que sirvam para criar partes e, até mesmo, objetos inteiros.

Nesse contexto, este trabalho teve o objetivo de avaliar a possibilidade do aproveitamento de resíduos de painéis de madeira, oriundos de indústrias moveleiras, na produção artesanal de pequenos objetos. Para isso, foram produzidos pequenos objetos com painéis e resíduos de painéis de madeira, para comparar a qualidade de seus atributos. Especificamente, os objetivos foram identificar quais tipos de painéis apresentam o melhor desempenho para os atributos avaliados; avaliar a qualidade dos objetos confeccionados com mistura de resíduos de painéis de madeira; e avaliar a viabilidade técnica da produção de pequenos objetos a partir de resíduos de painéis de madeira.

\section{MATERIAL E MÉTODOS}

Foi organizada uma coleção de 70 pequenos objetos, dos quais se escolheram 10, em razão da representatividade de classes de uso, como adornos, brinquedos e utilitários, e do grau de dificuldade de execução. Os objetos escolhidos foram: caixa de chá, caminhão, helicóptero, jacaré, porta-chaves, portaguardanapos, porta-ovos, porta-retrato, quebra-cabeça e suporte de vinho. O projeto gráfico e o aspecto final do porta-ovos, por exemplo, podem ser conferidos nas Figuras 1 e 2.

Foram elaborados os projetos gráficos dos 10 objetos no aplicativo AutoCAD, e escolhidos três artesãos para reproduzi-los. Cada um recebeu os projetos e os painéis de MDF, OSB e compensado e produziu 90 objetos, sendo 9 de cada. O total confeccionado foi de 270 objetos. O delineamento experimental foi montado em blocos casualizados, sendo considerados como blocos os artesãos e como fatores, os três materiais. Foram utilizadas três repetições de cada material.

Para os objetos confeccionados com resíduos de painéis, o delineamento experimental foi o inteiramente casualizado, em que os tratamentos utilizados foram os mesmos 10 objetos. Entretanto, os objetos foram confeccionados por um único artesão, o qual recebeu 
os resíduos de três empresas do polo moveleiro da cidade de Ubá, MG. Foram produzidas com esses resíduos três repetições de cada objeto, totalizando 30 objetos. Foi permitido que os materiais fossem misturados em um único objeto, que não receberia tratamento superficial, conforme a criatividade do artesão.
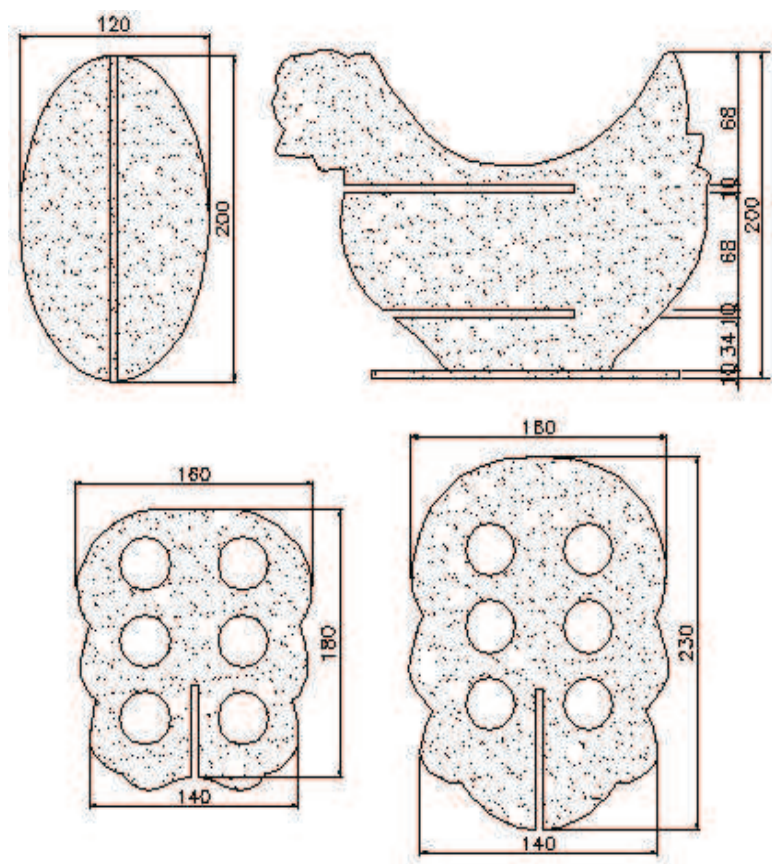

Figura 1 - Projeto gráfico do porta-ovos.

Figure 1-Graphic project of an egg holder.

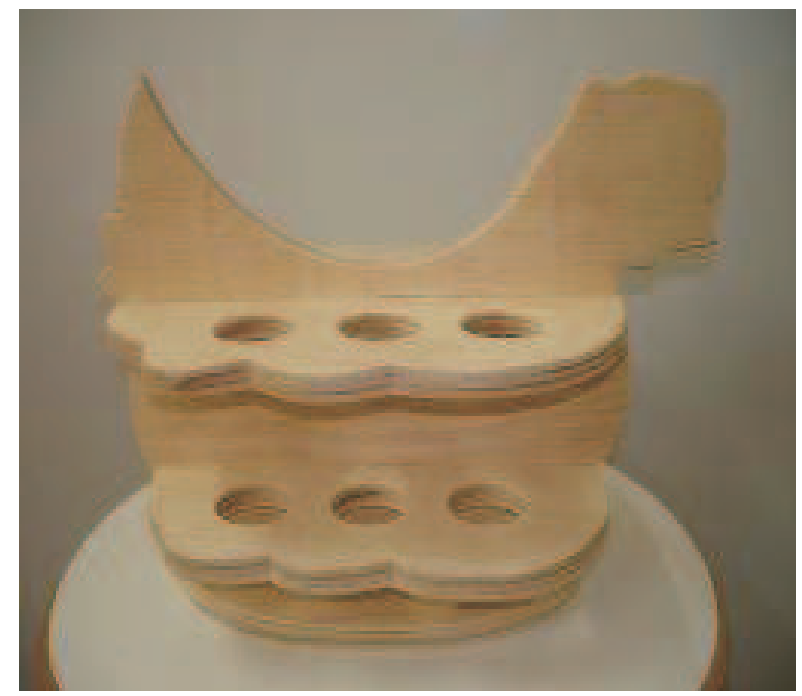

Figura 2 - Aspecto final do porta-ovos. Figure 2 - Final aspect of the egg holder.
Os objetos foram submetidos à avaliação subjetiva, realizada por quatro pessoas, sendo: um arquiteto, um consumidor, um marceneiro e um projetista. Apesar do caráter subjetivo dos atributos, os critérios que foram usados pelos avaliadores foram cuidadosamente predeterminados e discutidos entre eles.

Os atributos avaliados em cada objeto foram: acabamento, beleza, cor, funcionalidade, peso, proporção e simetria. Foram dadas a esses atributos notas de 0,5 (meio) a 5,0 (cinco), com incremento de 0,5. Ao contrário da forma adotada na norma ASTM D 1666-87, optouse por considerar a nota 0,5 como "péssima" e a nota 5,0 como "excelente", pois uma escala numérica decrescente em direção à "excelente" poderia confundir os avaliadores. Após a avaliação dos objetos, os dados foram processados no aplicativo SISVAR.

$\mathrm{Na}$ última etapa, foram comparadas as médias atribuídas aos objetos confeccionados com painéis e com resíduos de painéis, para cada atributo. O delineamento experimental utilizado foi em blocos casualizados, sendo considerados como blocos os objetos e, como tratamentos, os dois tipos de material (painéis e resíduos). Foram utilizadas 10 repetições.

\section{RESULTADOS E DISCUSSÃO}

\subsection{Objetos confeccionados a partir de painéis}

Por meio da análise de variância, observou-se que o efeito da interação material x objeto foi significativo para os atributos acabamento, cor e peso. Os atributos que não apresentaram interação foram beleza, funcionalidade, proporção e simetria, o que indica que, em determinado objeto, esses atributos não dependeram do material com o qual o objeto foi confeccionado. Qualquer um dos materiais avaliados (MDF, OSB ou compensado) imprimiu o mesmo padrão de beleza, de funcionalidade, de proporção e de simetria aos objetos.

Para o acabamento, quando se compararam os 10 objetos feitos com MDF, a avaliação atribuída aos objetos porta-chaves, porta-ovos e jacaré foi inferior à dos demais, mostrando que o MDF não resulta em um mesmo acabamento para todos os objetos. Aqueles três objetos apresentam bordas arredondadas, feitas em serra ticotico, ou detalhes construtivos que exigem muita precisão do artesão. Pelo fato de estar trabalhando com o MDF e, provavelmente, pensar que esse material facilita acabamentos, faltou aos artesãos esmero na confecção desses objetos.

R. Árvore, Viçosa-MG, v.33, n.1, p.171-177, 2009 
Nos 10 objetos produzidos em compensado, a qualidade de acabamento dos objetos suporte de vinho e helicóptero foi inferior à dos outros objetos. O compensado é um painel constituído de um conjunto de lâminas coladas com a grã alternada, geralmente em ângulo reto. Suas lâminas são, usualmente, coladas simetricamente a partir do centro ou miolo do painel (OLIVEIRA et al., 2005). É necessário destacar que a principal ferramenta de corte utilizada para a confecção dos objetos é uma serra de fita ou uma serra tico-tico que, ao cortar material laminado, nem sempre consegue acabamento superficial isento de rompimento das lâminas. Apesar disso, não se pode perder de vista que se trata de produtos artesanais, devendo ser valorizados mesmo quando apresentam pequenos defeitos.

Quanto à cor do objeto, o desenho da superfície e a rugosidade dos painéis influenciam a sua avaliação. O compensado apresentou, em média, o melhor desempenho, devido ao fato de manter o desenho da grã da madeira, o que, geralmente, é mais atraente e agrada às pessoas. Já a superfície rugosa e heterogênea do OSB, de textura mais grossa, provoca a dispersão da luz e, consequentemente, cria nuances de tonalidades diferentes, que podem não agradar aos avaliadores.

A beleza não é fácil de ser descrita, pois é uma questão de percepção subjetiva. É possível que 50\% dos objetos não tenham sido considerados belos pelo fato de não terem sido pintados e apresentarem sua textura natural. Ou por terem desagradado em outro atributo, já que a beleza de um objeto pode ser relacionada e influenciada por outros atributos.

Alguns objetos foram considerados mais funcionais que outros, o que pode ser justificado por sua versatilidade, que os torna muito viáveis economicamente, como é o caso do porta-ovos e da caixa de chá. O porta-ovos é uma peça útil e atrativa; a caixa de chá, por sua vez, é um objeto que apresenta versatilidade, podendo ser utilizada para outros fins.

\subsection{Objetos confeccionados a partir de resíduos de painéis}

Por meio da análise de variância, observou-se que apenas as avaliações para acabamento não apresentaram diferenças significativas entre os objetos, o que pode ser conferido nos valores das médias referentes aos atributos, na Tabela 1 . Esse resultado indica que qualquer um dos objetos pode ser confeccionado a partir de resíduos, atingindo-se um acabamento uniforme. A explicação para esse resultado se deve ao fato de cada objeto ter sido feito com materiais diversos, conforme a criatividade do artesão. Seguramente, ele escolheu, para uma parte mais complexa de determinado objeto, um resíduo que considerava mais fácil de trabalhar.

Para os outros atributos avaliados, houve diferença significativa entre os diferentes objetos. Pode-se supor que esses atributos sofram influência do material empregado em cada peça de um mesmo objeto, ou seja, pequena parte do objeto, confeccionada com material não agradável aos sentidos do avaliador, pode prejudicar a avaliação do conjunto do objeto.

Tabela 1 - Médias referentes aos atributos avaliados para objetos confeccionados com resíduos de painéis Table 1 -Means referring to the attributes evaluated for objects made with wood board residues

\begin{tabular}{|c|c|c|c|c|c|c|c|}
\hline \multirow[t]{2}{*}{ Objeto } & \multicolumn{7}{|c|}{ Atributos } \\
\hline & Acab & Beleza** & Cor & Funcio. & Peso & Prop. & Simet. \\
\hline Porta-guard. & $3,09 \mathrm{a}$ & $3,21 \mathrm{a}$ & $2,92 b$ & $5,00 \mathrm{a}$ & $4,46 b$ & $3,42 \mathrm{~d}$ & $3,38 \mathrm{~b}$ \\
\hline Suporte vinho & $2,92 \mathrm{a}$ & $3,13 \mathrm{a}$ & $3,42 b$ & $5,00 \mathrm{a}$ & $4,75 \mathrm{a}$ & $3,29 d$ & $3,58 \mathrm{~b}$ \\
\hline Porta-chaves & $2,79 \mathrm{a}$ & $3,17 \mathrm{a}$ & $3,42 b$ & $4,63 c$ & $4,25 c$ & $3,38 d$ & $2,58 \mathrm{c}$ \\
\hline Porta-ovos & $2,29 \mathrm{a}$ & $3,21 \mathrm{a}$ & $3,21 b$ & $4,88 \mathrm{~b}$ & $4,21 \mathrm{c}$ & $4,42 b$ & $3,04 \mathrm{c}$ \\
\hline Caixa de chá & $3,29 \mathrm{a}$ & $4,00 \mathrm{a}$ & $3,17 b$ & $5,00 \mathrm{a}$ & $4,75 a$ & $4,63 \mathrm{a}$ & $4,38^{\mathrm{a}}$ \\
\hline Porta-retrato & $3,04 \mathrm{a}$ & $3,54 \mathrm{a}$ & $4,00 \mathrm{a}$ & $5,00 \mathrm{a}$ & $4,00 \mathrm{c}$ & $4,75 \mathrm{a}$ & $3,38 b$ \\
\hline Jacaré & $2,75 \mathrm{a}$ & $3,42 \mathrm{a}$ & $3,63 b$ & $4,63 c$ & $4,88 \mathrm{a}$ & $4,63 \mathrm{a}$ & $2,83 \mathrm{c}$ \\
\hline Caminhão & $3,13 \mathrm{a}$ & $3,50 \mathrm{a}$ & $3,38 b$ & $5,00 \mathrm{a}$ & $4,54 b$ & $4,25 b$ & $3,33 b$ \\
\hline Helicóptero & $2,58 \mathrm{a}$ & $3,46 a$ & $3,46 b$ & $4,88 \mathrm{~b}$ & $4,13 \mathrm{c}$ & $4,00 \mathrm{c}$ & $3,88^{\mathrm{a}}$ \\
\hline Quebra-cabeça & $2,92 \mathrm{a}$ & $3,83 \mathrm{a}$ & $4,33 \mathrm{a}$ & $5,00 \mathrm{a}$ & $4,54 b$ & $4,79 a$ & $4,33^{\mathrm{a}}$ \\
\hline
\end{tabular}

Acab = acabamento; Funcio. = funcionalidade; Prop. = proporção; Simet. = simetria; Porta-guard. = porta-guardanapo.

* Médias seguidas de mesma letra no sentido da coluna não diferem entre si, a $5 \%$ de significância, pelo teste de Scott-Knott (exceto para o atributo Beleza).

** Para o atributo beleza, apesar de a análise de variância ter sido significativa a $5 \%$ de significância (p<0,0329), não foi detectada nenhuma diferença entre as médias pelos testes de Scott-Knott, Tukey e SNK.

R. Árvore, Viçosa-MG, v.33, n.1, p.171-177, 2009 


\subsection{Comparação entre objetos de painéis e objetos de resíduos}

Por meio da análise de variância da comparação, observou-se que as avaliações para os atributos acabamento, beleza e cor apresentaram diferenças significativas entre os objetos confeccionados com painéis e resíduos de painéis, conforme pode ser observado na Tabela 2. Quanto aos demais atributos, não houve diferença significativa entre os objetos produzidos com painéis e com resíduos.

Tabela 2 - Resumo da análise de variância de acabamento, beleza e cor dos objetos confeccionados com painéis e resíduos de painéis

Table 2 - Summary of the analysis of variance of finishing, beauty and color for objects made with panels and panel residues

\begin{tabular}{|c|c|c|c|c|}
\hline \multirow[t]{2}{*}{$\mathrm{FV}$} & \multirow[t]{2}{*}{ GL } & \multicolumn{3}{|c|}{ Quadrados Médios } \\
\hline & & Acabamento & Beleza & Cor \\
\hline Painéis/resíduos & 1 & $3,7758 * *$ & $1,8973 * *$ & $4,2136^{* *}$ \\
\hline Bloco & 9 & $0,1103 * *$ & $0,0978 \mathrm{~ns}$ & $0,0714 \mathrm{~ns}$ \\
\hline Erro & 9 & 0,0176 & 0,0573 & 0,1753 \\
\hline Total & 19 & & & \\
\hline CVexp & & 4,00 & 6,38 & 10,59 \\
\hline
\end{tabular}

**, * e ns: significativos a $1 \%$ e $5 \%$ de significância e não-significativo, respectivamente. $\mathrm{FV}=$ fonte de variação; $\mathrm{CV}=$ coeficiente de variação; e $\mathrm{GL}=$ graus de liberdade.
As médias referentes aos atributos analisados encontram-se na Tabela 3. Constata-se, nessa tabela, que houve diferença de padrão de acabamento para os objetos produzidos com painéis, com média de 3,75 , e aqueles confeccionados com resíduos, com média de 2,88 . A explicação para esse resultado se deve ao fato de, nos objetos produzidos com resíduos, vários tipos de painéis terem sido misturados, uns mais simples e outros mais complexos de serem usinados. Esse fato os deixou com acabamento menos homogêneo, o que não inviabiliza sua utilização na confecção de pequenos objetos.

Com relação aos atributos beleza e cor, também houve diferença significativa entre os objetos produzidos com painéis e os objetos produzidos com resíduos. Esses atributos sofreram influência da mistura de resíduos dentro do objeto, o que pode ter provocado sensação de conflito na mente do avaliador, prejudicando a classificação desses atributos, que tanto dependem da avaliação subjetiva pessoal.

Quando um objeto é produzido a partir de vários tipos de materiais, sua avaliação pode sofrer influência negativa de um material que não agrade ao avaliador, ou o consumidor. Um objeto produzido a partir de resíduos de mesmo material pode tornar a peça mais harmoniosa, com chances de atingir melhores resultados para os atributos acabamento, beleza e cor.

Tabela 3 - Médias referentes aos atributos dos objetos confeccionados com painéis e resíduos

Table 3 -Means referring to the attributes evaluated for objects made with panels and panel residues

\begin{tabular}{lccccccc}
\hline Material & \multicolumn{5}{c}{ Atributos } \\
\cline { 2 - 7 } & Acab. & Beleza & Cor & Funcio. & Peso & Prop. & Simet. \\
\hline Painéis & $3,75 \mathrm{a}$ & $4,06 \mathrm{a}$ & $4,41 \mathrm{a}$ & $4,90 \mathrm{a}$ & $4,67 \mathrm{a}$ & $4,39 \mathrm{a}$ & $4,22^{\mathrm{a}}$ \\
Resíduos & $2,88 \mathrm{~b}$ & $3,45 \mathrm{~b}$ & $3,49 \mathrm{~b}$ & $4,56 \mathrm{a}$ & $4,45 \mathrm{a}$ & $4,16 \mathrm{a}$ & $3,97^{\mathrm{a}}$ \\
\hline
\end{tabular}

Acab. = acabamento; Funcio. $=$ funcionalidade; Prop. $=$ proporção; e Simet. $=$ simetria.

* Médias seguidas de mesma letra no sentido da coluna não diferem entre si, a 5\% de significância, pelo teste de F.

\section{CONCLUSÕES}

De acordo com os resultados, conclui-se que todos os painéis de madeira foram adequados para a confecção dos 10 objetos selecionados. Alguns painéis se destacaram em relação a outros, conforme o atributo avaliado. Todos os objetos confeccionados com mistura de resíduos de painéis alcançaram acabamento uniforme; a mistura pode influenciar negativamente a avaliação dos atributos acabamento, beleza e cor do objeto. Os objetos confeccionados com mistura de resíduos de painéis de madeira não apresentaram diferenças significativas em relação aos produzidos com painéis em relação aos atributos funcionalidade, peso, proporção e simetria.

Assim, é tecnicamente viável a produção de pequenos objetos a partir de resíduos de painéis de madeira oriundos de indústrias moveleiras.

\section{AGRADECIMENTOS}

Ao Curso de Pós-Graduação em Engenharia Florestal do Departamento de Ciências Florestais da Universidade Federal de Lavras, à FAPEMIG e ao CNPq, pelo apoio à realização deste trabalho.

R. Árvore, Viçosa-MG, v.33, n.1, p.171-177, 2009 


\section{REFERÊNCIAS}

\section{AMERICAN SOCIETY FOR TESTING AND} MATERIALS - ASTM. ASTM D 1666-87

(Reapproved 1994). Standard Method for Conducting Machining Tests of Wood and WoodBase Materials. Philaldelphia, 1995. p.226-245.

BRAND, M. A. et al. Avaliação do processo produtivo de uma indústria de manufatura de painéis por meio do balanço de material e do rendimento da matéria-prima. Revista Árvore, v.28, n.4, p.553-562, 2004.
OLIVEIRA, A. D.; RIBEIRO, I. S. A.; SCOLFORO, J. R. S. Análise do mercado internacional de compensado. Revista Árvore, v.29, n.2, p.311-320, 2005.

STERnAdt, G. H. Pequenos objetos de madeira - POM, compostagem de serragem de madeira. Brasília: LPF, 2002. 29p.

TOMASELLI, I.; DELESPINASSE, B. A Indústria de painéis do Brasil. A tendência de mudança no perfil da produção brasileira. STCP Informativo, n.1, p.17-20, 1997. 